\title{
Understanding GFP Post-Translational Chemistry: Structures of Designed Variants that Achieve Backbone Fragmentation, Hydrolysis and Decarboxylation
}

David P. Barondeau, Carey J. Kassmann, John A. Tainer, and Elizabeth D. Getzoff ${ }^{\star}$

Department of Molecular Biology, The Skaggs Institute for Chemical Biology, The Scripps Research Institute, 10550 N.

Torrey Pines Rd., La Jolla 92037

\section{Supporting Information}

Here we include a SDS-gel (Fig. S1) that shows the denaturation and native backbone cleavage products, a structural overlay of precursor and mature chromophore states with the GAG F64L and GSG F64L variants (Fig. S2), and a structural overlay of ASG and GSG variants with and without the F64L substitution (Fig. S3).

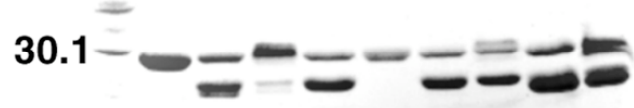

\section{4}

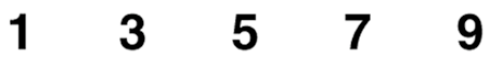

A
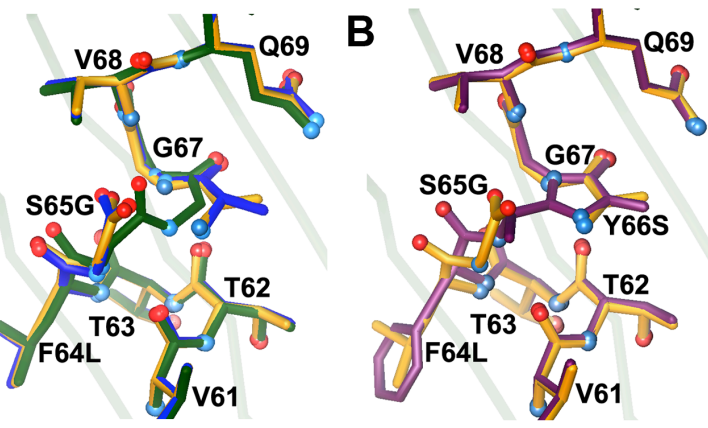

Figure S2. Structural overlay of ASG and GSG variants with and without the F64L substitution. (A) Overlay of ASG F64 (yellow) and ASG F64L (green) showing the intact MIO ring and small shift induced by the F64L substitution. (B) Overlay of GSG F64 (green) and GSG F64L (blue) emphasizing the S65G Y66S spontaneous peptide hydrolysis and significant backbone shift of residue S65G due to the decarboxylation of the GSG F64 variant (green).

Figure S1. GFP backbone cleavage by denaturation-induced backbone fragmentation and spontaneous peptide hydrolysis. SDSgel results showing GFP clevage: Lane 1-9 are (1) GFPsol, (2) ASG F64, (3) ASG F64 (not boiled), (4) GGG, (5) ASG R96A, (6) GAG F64L, (7) GAG F64L (not boiled), (8) ASG F64 H148G, (9) ASG F64 H148G (not boiled). All samples contained 4 M urea. Molecular weight standards are in the left-most lane.

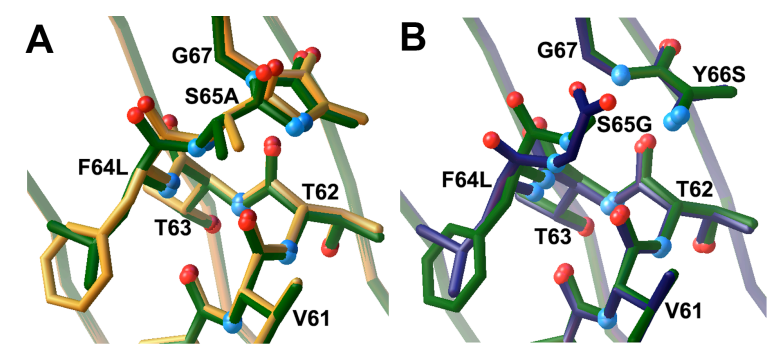

Figure S3. Structural overlay of precursor and mature chromophore states with cleaved variants. A) Overlay of the backbone-cleaved GAG F64L (blue) and GSG F64L (yellow) variants with the GFP GGG precyclized variant (green). B) Superposition of the backbone cleaved GSG F64L (yellow) and stable ring-forming ASG F64 (purple) variant. 